\title{
Developing adaptive control: Age-related differences in task choices and awareness of proactive and reactive control demands
}

\author{
J. C. Niebaum ${ }^{1}$ (D) N. Chevalier ${ }^{2} \cdot$ R. M. Guild ${ }^{3} \cdot$ Y. Munakata ${ }^{1}$
}

Accepted: 9 September 2020 / Published online: 2 October 2020

(C) The Psychonomic Society, Inc. 2020

\begin{abstract}
Developmental changes in executive function are often explained in terms of core cognitive processes and associated neural substrates. For example, younger children tend to engage control reactively in the moment as needed, whereas older children increasingly engage control proactively, in anticipation of needing it. Such developments may reflect increasing capacities for active maintenance dependent upon dorsolateral prefrontal cortex. However, younger children will engage proactive control when reactive control is made more difficult, suggesting that developmental changes may also reflect decisions about whether to engage control, and how. We tested awareness of temporal control demands and associated task choices in 5-year-olds and 10year-olds and adults using a demand selection task. Participants chose between one task that enabled proactive control and another task that enabled reactive control. Adults reported awareness of these different control demands and preferentially played the proactive task option. Ten-year-olds reported awareness of control demands but selected task options at chance. Five-yearolds showed neither awareness nor task preference, but a subsample who exhibited awareness of control demands preferentially played the reactive task option, mirroring their typical control mode. Thus, developmental improvements in executive function may in part reflect better awareness of cognitive demands and adaptive behavior, which may in turn reflect changes in dorsal anterior cingulate in signaling task demands to lateral prefrontal cortex.
\end{abstract}

Keywords Development $\cdot$ Cognitive control $\cdot$ Decision-making

Cognitive control, the ability to coordinate thoughts and behaviors to accomplish goals, improves dramatically across childhood (Davidson, Amso, Anderson, \& Diamond, 2006; Prencipe et al., 2011; Zelazo \& Carlson, 2012). For example, children transition from primarily engaging control reactively, recruiting control as needed, to engaging control proactively, in anticipation of need, as they age (Chatham, Frank, \& Munakata, 2009; Gonthier, Zira, Colé, \& Blaye, 2019;

Electronic supplementary material The online version of this article (https://doi.org/10.3758/s13415-020-00832-2) contains supplementary material, which is available to authorized users.

J. C. Niebaum

jcniebaum@ucdavis.edu

1 Department of Psychology and Center for Mind and Brain, University of California-Davis, Davis, CA, USA

2 School of Philosophy, Psychology, and Language Sciences, University of Edinburgh, Edinburgh, UK

3 Department of Psychology and Neuroscience, University of Colorado Boulder, Boulder, CO, USA
Lucenet \& Blaye, 2014). These improvements support children's behavior through an improving ability to keep information and goals in mind (Best, Miller, \& Naglieri, 2011; Blair \& Razza, 2007; Carlson \& Wang, 2007; Cartwright, 2012), and children's cognitive control predicts important concurrent and future outcomes, such as academic achievement, health, and income (Ahmed, Tang, Waters, \& Davis-Kean, 2019; Moffitt et al., 2011; Robson, Allen, \& Howard, 2020).

Extensive research efforts have focused on understanding improvements in core cognitive processes, such as working memory, that might support the increased use of proactive control (e.g., Davidson et al., 2006; Munakata, Snyder, \& Chatham, 2012). For example, increases in working memory capacity support the transition to proactive control as children age (Gathercole, Pickering, Ambridge, \& Wearing, 2004; Troller-Renfree, Buzzell, \& Fox, 2020). Such improvements have been linked to maturation of lateral prefrontal cortex (IPFC) and its increasing connectivity with other brain regions, including dorsal anterior cingulate cortex and striatum, that continue to emerge from young childhood into adulthood (Andrews-Hanna et al., 2011; Buss \& Spencer, 2018; Ezekiel, Bosma, \& Morton, 2013; Fiske \& Holmboe, 2019; Lopez, 
Kandala, Marek, \& Barch, 2019; Luna, Padmanabhan, \& O'Hearn, 2010; Vink et al., 2014). 1PFC is thought to support the flexible updating and maintenance of task rules (Koechlin \& Summerfield, 2007; Niendam et al., 2012; Wendelken, Munakata, Baym, Souza, \& Bunge, 2012). Thus, developmental changes like the transition to increasingly proactive control may reflect increasing capacities for active maintenance dependent upon IPFC.

However, children must also become adept at coordinating appropriate control strategies, known as metacontrol, given their goals and environmental demands (Chevalier, 2015). Five-yearold children, who tend to engage control reactively, will engage control proactively when reactive control is made more difficult by removing a sorting rule prior to the target to be sorted, exhibiting both faster response times and pupillometric and ERP markers of proactive control (Chevalier, Martis, Curran, \& Munakata, 2015). In contrast, older children will engage control proactively when possible and implement control reactively only when proactive preparation is prevented. Thus, younger children can engage proactive control but differ from older children and adults in the contexts in which they do so. Age-related improvements in cognitive control may thus reflect improvements in not only core cognitive processes but also in improved metacontrol to adaptively select what type of control to engage, when to do so, and the kinds of tasks to take on.

Children's awareness of control demands and adaptive task selection has been investigated using a demand selection task (Niebaum, Chevalier, Guild, \& Munakata, 2019), in which participants chose between one task that switched between sorting rules more frequently than another, resulting in greater control demand (Monsell, 2003). Adults, 11-year-olds, and 6year-olds were all slower and less accurate on rule switch trials and thus had demand signals to potentially use to select the easier task option. However, only 11-year-olds and adults reported awareness of these different control demand and preferentially selected the option with fewer rule switches (Niebaum, et al., 2019), supporting prior work in only adults (Kool, McGuire, Rosen, \& Botvinick, 2010; cf. Gold et al., 2015). In contrast, 6-year-olds were unaware of demand differences and selected tasks at chance.

What leads to these age-based differences? Young children may be less sensitive to task switching demands than older children and adults but more attuned to other developmentally relevant control signals. For example, due to age-related biases in engaging proactive and reactive control, younger children may be more sensitive to temporal control demands compared with other control demand signals, like task switching demands, and select tasks enabling reactive control, their preferred control mode. Additionally, signals of demand may be different across age groups in different domains. For example, the relative benefits of proactive control may increase with age, which could result in differences in task choices and awareness of task demands across development.
We examined whether task choices and awareness of proactive and reactive control demands differ across development. Adults and 5-year-old and 10-year-old children completed a demand selection task presenting two task options that encouraged either proactive or reactive control. Participants were asked to sort pictures from two card decks that differed in the temporal presentation of a sorting rule. One deck, the proactive deck, displayed the sorting rule before each picture, allowing participants to prepare for a sorting dimension, and occluded the rule during target presentation. The other deck, the reactive deck, presented the sorting rule and picture simultaneously, preventing such preparation. After being familiarized with both decks, participants were able to choose which deck to play.

We predicted that the proportion of proactive deck selections would increase with age. Because 5-year-olds tend to engage control reactively, we expected 5-year-olds to preferentially select the reactive deck if exhibiting awareness of the temporal control differences between decks. We expected 10year-olds to preferentially play the proactive deck; however, 10 -year-olds may also select decks at chance because 10 -yearolds have been shown to use relative accuracy differences to select tasks but have not shown accuracy benefits with proactive control engagement (Chevalier et al., 2015; Niebaum et al., 2019). Because adults tend to engage control proactively and have previously been shown to prioritize relative response time efficiency signals to select tasks (Kool et al., 2010; Niebaum et al., 2019), we expected adults to select the proactive deck. Thus, we also expected that relative accuracy differences between decks would predict deck selections in 10 -year-olds and that relative efficiency benefits for the proactive deck would predict deck selections in adults. Finally, we predicted that awareness of deck differences, subjective deck preferences, and awareness of performance differences between decks would change with age.

\section{Method}

\section{Participants}

We analyzed a sample of 42 5-year-olds (5yo: $M=5.60$, range: 5.07-6.09, 21 males), 40 10-year-olds (10yo: $M=$ 10.59 years, range: $10.07-11.02,23$ males), and 75 adult participants $(M=20.22$, range: $17.96-38.50$, three not reporting, 33 males). We selected these ages to match prior work on the implicit coordination of proactive and reactive control to task demands and include adults for further comparison (Chevalier et al., 2015). Children transition from primarily engaging control reactive to engaging control proactively at about 6 years of age (Lucenet \& Blaye, 2014); thus, we included young children biased towards implementing reactive control to contrast with older, typically more proactive children and adults. No 
upper age limit was used for adults because we had no hypotheses about changes in proactive deck selections after reaching adulthood. Five additional 5 yo quit the study session prior to completion, one additional 10yo was excluded due to a parent describing deck differences during the study session, and three additional adults were missing behavioral data from the demand selection task due to program errors.

Our effect size estimate was based on the average effect size of two adult samples completing similar paradigms in pilot samples, in which the proportion of proactive deck selections, our primary outcome, was tested against a hypothetical sample of $50 \%$ proactive deck selections, indicating no preference for either the proactive or reactive deck (the upper range of our 5yo group prediction) with similar standard deviation. $\mathrm{G}^{*}$ Power 3.1 indicated that 36 participants per cell were needed detect a Cohen's $d=.7$ at $90 \%$ power at an alpha of .05 using a traditional analysis of variance (ANOVA) because guidelines for conducting power analyses for KruskalWallis tests have not been established (Faul, Erdfelder, Lang, \& Buchner, 2007; McDonald, 2014). Because we anticipated non-normal distributions in the primary outcome variable and sought to increase power for additional analyses, we recruited at least 40 participants per group. Child participants were recruited until reaching the minimum sample completing the demand selection task. As child data collection typically requires more time, we continued to recruit adults to increase statistical power because our primary preregistered statistical tests are robust to differences in group size (McHugh, 2013; Meyer \& Seaman, 2013).

Child participants were recruited from the participant database of the Cognitive Development Center maintained at the University of Colorado Boulder. Informed consent was obtained from legal parents/guardians, and child assent (verbal and/or written) was also obtained. Parents/guardians received minimal monetary compensation for travel costs, and child participants received a token for study participation. Adult participants were recruited from the Department of Psychology and Neuroscience subject pool at the University of Colorado Boulder for partial course credit. Written informed consent was obtained prior to participation. Participants were tested at the Cognitive Development Center at the University of Colorado Boulder, and the local Institutional Review Board approved all study procedures.

\section{Demand selection task}

The demand selection task was analogous to Niebaum et al. (2019) and programmed in PsychoPy v2.82 (Peirce et al., 2019). Critically, the decks differed in the temporal presentation of the sorting rule. For one deck, the proactive deck, the sorting rule was presented 1.5 seconds before the target and then occluded with a grey square when the target appeared, encouraging proactive control. For the other deck, the reactive deck, a grey square was presented in place of the sorting rule for 1.5 seconds, and then, the sorting rule and target were presented simultaneously, preventing proactive control. Participants were not notified of any differences between decks.

The task typically took 20-30 min and comprised three phases of rule practice, baseline deck familiarization, and deck choice (see Fig. 1). In all phases, participants were asked to sort pictures (i.e., "targets") according to their color or shape. Four targets were used (orange or green circle or triangle). Response buttons were identified via two multidimensional pictures (e.g., an orange triangle to indicate orange and triangle responses and a green circle to indicate green and circle responses) displayed on the bottom left and right of the screen and also presented on the response pad horizontally above the response buttons. Deck choice buttons were identified via two blue boxes above two buttons to the left or right of the target response buttons. Participants saw a smiley face and heard a positive sound after correct responses and a frowning face and negative sound after incorrect responses. After each correct trial, participants were given a piece of digital candy shown at the bottom right of the screen; a candy piece was removed after incorrect trials. We did not set an upper latency bound for positive feedback to prevent participants from selecting the proactive deck to avoid negative feedback from long response latencies, which is consistent with similar paradigms using these trial structures (Chevalier et al., 2015; Doebel et al., 2017). The candy count enabled long-term tracking of general performance and along with the positive audio feedback, helped to provided continued motivation for participants to perform well throughout the task, similar to other childfriendly task-switching paradigms (Chevalier, Dauvier, \& Blaye, 2018; Chevalier et al., 2015; Niebaum et al., 2019).

\section{Rule practice phase}

Each sorting rule was explained in turn, followed by four practice trials with each rule and four mixed rule practice trials. Each set of practice trials was repeated until participants answered all four trials for each rule and the mixed rule practice correctly, and participants were instructed to respond to the target according to the cued rule as quickly and accurately as possible. For all practice trials, the sorting rule was displayed 1.5 seconds prior to the target and remained on screen during target presentation to prevent biasing participants towards implementing proactive or reactive control during rule practice.

\section{Deck familiarization phase}

Participants were then told that the pictures would be drawn from two blue card decks on the upper left and right of the screen and instructed to continue to respond as quickly and accurately as possible and pay attention to which deck the pictures came from. Blue cards transitioned to the center of 
the computer screen and were flipped when reaching the center. Participants completed 60 baseline trials (30 trials/deck) divided into 15-trial blocks. Proactive deck placement (left or right side) was counterbalanced across participants, and each deck presented the same series of rule switches and repeats during familiarization.

\section{Deck choice practice phase}

Participants then practiced choosing both the left and right decks for five trials each. Right and left deck selections were made with two response buttons on the outside of the target response buttons and indicated above the response buttons with two blue rectangles. Prior to each trial, participants were told to fixate on a plus sign between the decks. A question mark appeared in place of the plus sign to indicate that participants could choose which deck to play. After selection, cards transitioned to the center of the screen and then revealed cues and targets.

\section{Deck choice phase}

After deck choice practice, participants were informed that they could choose whichever deck they preferred to play after every trial, that they were free to switch decks whenever they wanted, and that if they began to prefer one deck more than the other, they could play that deck more often or even all the time. Participants then completed 50 free-choice trials divided across two blocks.

\section{Posttask questionnaire}

After the demand selection task, the experimenter read aloud six questions to all participants to assess awareness of deck differences and subjective experiences with each deck. Participants responded verbally, and responses were recorded on paper by the experiment. Participants responded with the left or right deck for all questions except for the initial question, to which participants responded yes or no. Responses were later recoded as the proactive or reactive deck. The questions were as follows: Were there any differences between decks?; Did you like one deck more than the other?; Was one deck easier than the other?; Were you faster on one deck more than the other?; Did you get more right on one deck than the other?; and Did one deck make you think harder than the other?. If participants did not report a deck, the experimenter asked the question again, prefaced with the phrase, "If you had to choose ...." For analyses regarding responses to these questions, initial and forced choice responses were collapsed. ${ }^{1}$

\footnotetext{
${ }^{1}$ We also made a post hoc decision to exclude participants who refused to answer a question in analyses assessing whether age groups significantly differed from chance responding on the question. No more than one participant refused to answer any specific question.
}

Additionally, participants gave open responses to each question explaining their answer. Analyses of the free response data are included in the Supplementary Materials.

\section{Statistical analyses}

This project was preregistered with the Open Science Framework (OSF; osf.io/ung52/), and analyses were conducted as proposed unless otherwise noted. All data and materials are also available at the project's OSF page. For each participant, mean response time for correct trials was calculated after removing outliers, defined as the mean plus three standard deviations and less than $200 \mathrm{~ms}$, or mean minus three standard deviations (1.98\% of trials removed), in accordance with Chevalier et al. (2015). Because response times were skewed on both proactive and reactive decks in all age groups during familiarization (all $p \mathrm{~s}<.01$ ), response times for each deck were log-transformed to reduce skew for correlations and to better meet assumptions for the ANOVA for task performance (Meiran, 1996).

Proactive deck preference was defined as the proportion of choice trials in which participants selected to play the proactive deck. As predicted, the proportions of proactive deck selections were not normally distributed (Shapiro-Wilk normality test: overall $p \mathrm{~s}<.001$; all group $p \mathrm{~s}<.05$ ); thus, a Kruskal-Wallis test was used to test for group differences in the proportion of proactive deck selections, and Wilcoxon signed-rank tests against chance deck selection were used to determine deck preference. Follow-up exploratory hierarchical models were conducted to examine age-related differences in deck selections across the choice phase and whether awareness of deck differences differentially influenced deck selections across the choice phase according to age. To ascertain performance metrics of relative demands, we calculated mean differences in response time and accuracy between the proactive and reactive decks for each participant. ${ }^{2}$ Chi-square tests were conducted to test age group differences in binary responses to posttask questions, and single proportion tests were conducted within groups to determine whether responses significantly differed from chance responding. All analyses were performed with the open-source R software (RStudio Team, 2015). Bayesian analyses were conducted with the BayesFactor package (Morey, Rouder, Jamil, \& Morey, 2015), and data were visualized using the ggplot2 package in $\mathrm{R}$ (Wickham, 2016).

\footnotetext{
${ }^{2}$ Age groups may differ in their weighting of speed-accuracy trade-offs between the two decks, which may influence perceptions of demand for each task. We did not have a priori hypotheses regarding age differences in these trade-offs across tasks, and this issue may be challenging to test (e.g., given low reliability of condition contrasts from drift diffusion model parameters; Enkavi et al., 2019), but could be explored in future work.
} 
(a) Rule Practice

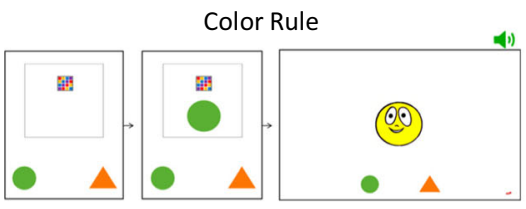

(b) Baseline Deck Familiarization Phase

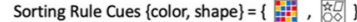

Targets $=\{\mathbf{A}, \boldsymbol{O}, \boldsymbol{\Delta}, \boldsymbol{0}\}$

Feedback $=\{(20$, , 장, $, \infty, 4(1),(1)\}$

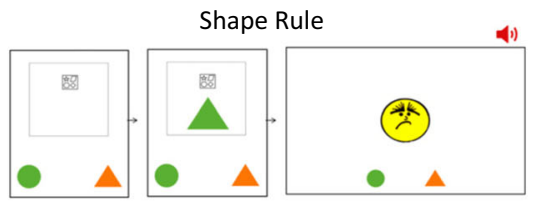

Reactive Trial

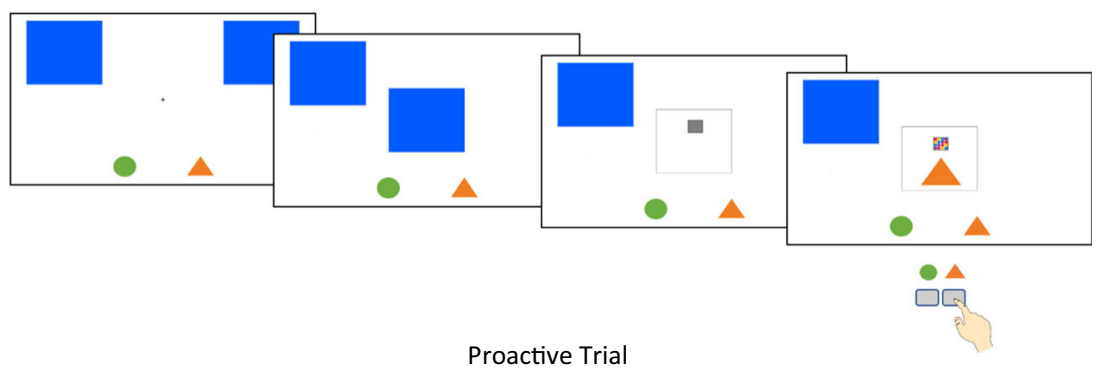

Proactive Trial

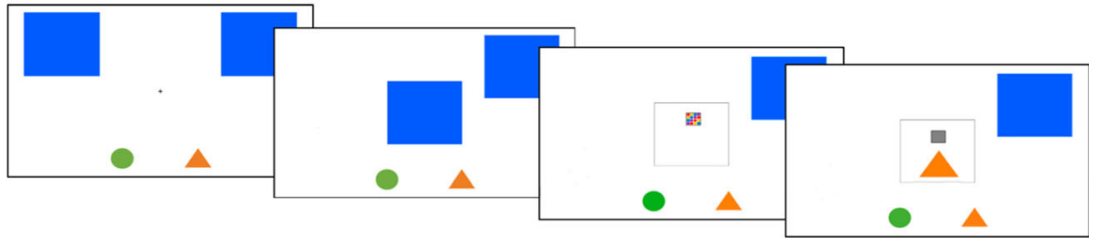

- 1

(c) Deck Choice Phase

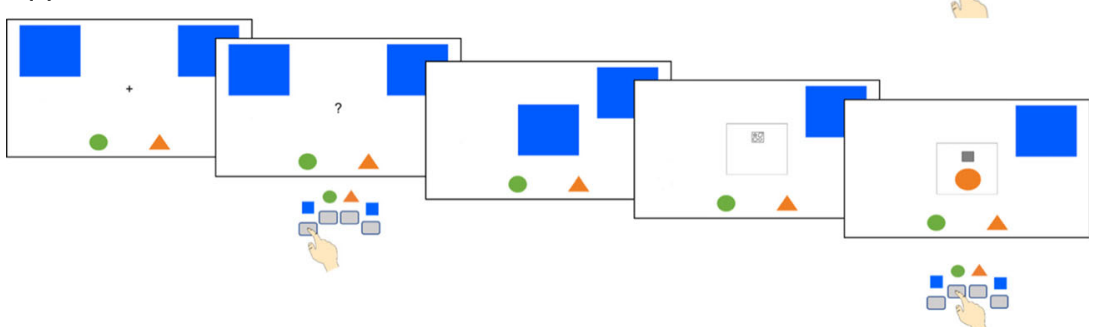

Fig. 1 The demand selection task flow. a Rule practice phase: Participants practiced sorting by the shape and color sorting rules in isolation (four trials/rule) and then together (four trials). The rules were presented prior to the probe and remained visible for the entire trial. The final frame presents positive and negative trial feedback. Small digital candy was given or removed for correct and incorrect responses throughout the task at the bottom right of the screen. b Familiarization phase (60 trials): Participants were familiarized with each of the two card decks. For the reactive trial deck, the right deck moved to the center of the screen and then flipped. A grey square occluded the sorting rule $(1.5 \mathrm{~s})$, and the sorting rule and target are then presented simultaneously. For proactive trials, the rule is presented prior to the target $(1.5 \mathrm{~s})$, and then removed when the target appears. c Deck choice phase (50 trials): Participants selected which deck to play every trial by pressing the far left or far right button on the response pad (underneath blue squares) and then responded as before using the middle two buttons. (Color figure online)

\section{Results}

\section{Rule practice performance}

Age groups differed in the number of times participants needed to repeat a practice run to achieve $100 \%$ accuracy, $F(2,154)=$ $39.07, p<.001$. Additional rule practice was not correlated with familiarization phase accuracy in either child group (5yo: $r=$

$-.17,[-.45, .14], p=.27 ; 10$ yo: $r=-.13,[-.42, .19], p=.44)$, indicating that additional practice did not confer a performance benefit. In adults, practice run repeats significantly negatively correlated with familiarization phase accuracy in the adult groups $(r=-.42[-.59,-.22], p<.001)$, indicating that adults who performed poorly in practice continued to perform poorly later in the task. Requiring all participants to meet the practice criteria to proceed ensured that all participants understood all rules. 


\section{Deck familiarization phase performance}

Age groups differed in overall accuracy, $F(2,154)=16.44, p$ $<.001$, overall $\log \mathrm{RT}, F(2,154)=239.41, p<.001$, proactive deck accuracy, $F(2,154)=31.34, p<.001$, proactive deck RT, $F(2,154)=108.86, p<.001$, and reactive deck RT, $F(2$, $154)=81.52, p<.001$. Age differences for reactive deck accuracy were marginal, $F(2,154)=2.73, p=.069$, likely due to the high accuracy across groups. We focus here on differences in accuracy and response time between the proactive and reactive decks, our preregistered indices of relative task demands. As predicted, all groups were significantly faster on the proactive than the reactive deck (adults: $M=$ $0.46, S D=.19), t(74)=20.70, p<.001$; (10yo: $M=0.37$, $S D=.17), t(39)=13.55, p<.001 ;(5$ уо: $M=0.21, S D=.22)$, $t(41)=6.29, p<.001$, and these correct log RT differences between decks also differed between age groups, $F(2,154)=$ $21.34, p<.001$. Follow-up Tukey's HSD were used to test all pairwise group comparisons to determine whether specific age groups differed in their relative performance between decks; differences in response times between the proactive and reactive decks was marginally larger for adults than 10yo (adjusted $p=.06$ ) and significantly larger than 5yo (adjusted $p<$ .001 ) and larger for 10yo than 5yo (adjusted $p<.001$ ). These results suggest that the differences in response efficiency when playing the proactive deck compared with the reactive deck increased with age. As predicted, adults and 10yo showed no accuracy differences between decks (adults: $M=$ $-0.53 \%), t(74)=-0.69, p=.49 ;(10 \mathrm{yo}: M=0.08 \%), t(39)=$ $0.07, p=.95$, whereas 5 yo were significantly more accurate on the reactive deck than the proactive deck $(M=6.19 \%)$, $t(41)=4.55, p<.001$. These relative accuracy differences differed between age groups, $F(2,154)=10.98, p<.001$, driven by significant differences between the 5 yo and both older age groups (Tukey's HSD, adjusted $p$ s $<.01$ ). Thus, although 5yo responded faster on the proactive deck than the reactive deck, they were less accurate on the proactive deck. In contrast, the adults and 10yo were faster on the proactive deck but showed no decreases in accuracy. Descriptive performance statistics are presented in Table 1. Post hoc exclusion of four outliers in the 10yo group (one outlier in accuracy differences and three outliers in response time between decks) did not change most results, and thus, all participants are included in the reported analyses. Further details on these analyses are available in the Supplementary Materials.

\section{Selection of proactive and reactive decks}

Age groups differed in the proportion of proactive deck selections (Kruskal-Wallis chi-squared $=18.61, p<.001$ ). Adults selected the proactive deck more than 10yo $(p<.01)$ and 5 yo $(p<.001)$, and 10yo and 5yo did not significantly differ $(p=$ $.24)$, as indicated by follow-up pairwise comparisons using the Wilcoxon rank sum test with Holm-Bonferroni adjusted $p$ values. As predicted, adults selected the proactive deck significantly more often than chance $(M=70.27 \%, S D=31.62, p$ $<.001$ ), whereas the 10 yo and 5 yo did not significantly differ from chance (10yo: $M=50.65 \%, S D=32.49, p=.96 ; 5$ yo: $M$ $=41.29 \%, S D=35.77, p=.19$; see Fig. 2).

We also conducted exploratory analyses with a linear code for age group and with age in days as continuous predictors of proactive deck selections. Similar results were obtained using a linear code for age group $(B=0.15, t=4.725, p<.001)$, and age positively correlated with proportion of proactive deck selections $(r=.39,[.23, .51], t=5.76, p<.001)$. Collectively, these results indicate that the proportion of proactive deck selections increased with age, confirming our primary prediction.

We also conducted exploratory hierarchical logistic regressions at the trial level. We predicted proactive deck selections with trial number, age group (using a linear code with 5-yearolds coded as 0 ), and their interaction, with random intercepts and trial slopes for participants. We observed main effects of age group $(B=0.24, z=2.87, p<.01)$ and trial number $(B=-0.07, \mathrm{z}$ $=-3.76, p<.001)$, as well as a significant group by trial interaction $(B=0.04, z=4.75, p<.001)$. Proactive deck selections increased with age and decreased across trials on average, but changes across trials varied by age group. Specifically, proactive deck selections increased across trials in adults $(r=.70,[0.52$, $\left..82], p<.001, \mathrm{BF}_{10}=4.65 \times 10^{5}\right)$, showed no significant change across trials in 10yo $\left(r=-0.14,[-0.40, .14], p=.33, \mathrm{BF}_{10}=.49\right)$, and decreased across trials in 5 yo $(r=-0.46[-0.66,-0.22], p<$ $.001, \mathrm{BF}_{10}=60.84$ ), as revealed by follow-up exploratory correlation tests (see Fig. 3).

\section{Associations between performance during familiarization and deck selections}

We conducted simple correlations between our performance metrics of relative demand, specifically response time and accuracy differences between decks, and the proportion of proactive deck selections in each age group. No correlations were observed between relative speed differences between decks and subsequent proportions of proactive deck selections for any age group (see Fig. 4, all $p$ s $>$.15). Similarly, no correlations were observed between relative accuracy differences between decks and subsequent proportions of proactive deck selections for any age group (see Fig. 4, all $p \mathrm{~s}>$.18). Additionally, we ran Bayesian analyses to determine whether the evidence favored the null hypothesis of no relationship between performance metrics and proportion of deck selections or the alternative. Bayes factors for all correlations provided anecdotal evidence in favor of the null hypothesis (all BFs < 1) (Makowski, Ben-Shachar, \& Lüdecke, 2019). 
Table 1. Deck familiarization performance across age groups

\begin{tabular}{llll}
\hline & 5-year-olds & 10-year-olds & Adults \\
\hline Overall accuracy** & $88.21 \%(8.05)$ & $92.13 \%(5.39)$ & $94.71 \%(4.56)$ \\
Overall log response time** & $8.04(0.47)$ & $7.20(0.22)$ & $6.78(0.20)$ \\
Proactive deck accuracy** & $85.16 \%(8.81)$ & $92.08 \%(5.53)$ & $94.98 \%(5.24)$ \\
Reactive deck accuracy* & $91.27 \%(9.51)$ & $92.17 \%(7.76)$ & $94.44 \%(6.04)$ \\
Relative accuracy difference** & $-6.11 \%(8.77)^{\wedge}$ & $0.08 \%(8.08)$ & $0.53 \%(6.67)$ \\
Proactive deck log response time** & $7.94(0.49)$ & $7.02(0.26)$ & $6.55(0.25)$ \\
Reactive deck log response time** & $8.15(0.47)$ & $7.39(0.22)$ & $7.01(0.19)$ \\
Relative response time difference** & $0.21(0.22)^{\wedge}$ & $0.37(0.17)^{\wedge}$ & $0.46(0.19)^{\wedge}$ \\
\hline
\end{tabular}

Note. Data are presented as means $(S D)$. Response times are log-transformed from mean millisecond response times for each participant. Age groups differed in overall accuracy and response time, as well as in accuracy and response time differences between proactive and reactive decks: relative response time differences between decks increased with age, and 5-year-olds had greater accuracy differences between decks compared with the older age groups. $*$ indicates a trend group difference $(p=.067)$. $* *$ indicates group differences at $p<.001{ }^{\wedge}{ }^{\wedge}$ indicates differences from 0 at $p<.001$

\section{Subjective awareness and preferences for proactive and reactive decks}

Age groups differed in their responses to all 6 posttask questions about the decks. Binary responses to the posttask questions are included in Table 2. First, age groups differed in reporting whether there were any differences between the decks $\left(\chi^{2}=23.20, p<.001\right)$. Adults and 10yo responded that there were deck differences more frequently than chance (adults: $86.49 \%, \chi^{2}=37.96, p<.001 ; 10$ yo: $82.5 \%, \chi^{2}=$ $15.63, p<.001)$, whereas 5 yo responded at chance levels (47.62\%, $\left.\chi^{2}=0.02, p=.88\right)$.

Although adults and older children thus differed from younger children in reporting whether there were any differences between decks, adults differed from both older and younger children on questions about subjective experiences and performance on the decks. Adults strongly preferred the proactive deck on posttask questions, whereas older children showed no significant leanings. Younger children preferentially reported the reactive deck on only some questions. On questions about subjective experiences with the decks, age groups differed in which deck was preferred $\left(\chi^{2}=21.97, p\right.$ $<.001)$, easier $\left(\chi^{2}=34.25, p<.001\right)$, and required more cognitive effort $\left(\chi^{2}=17.78, p<.001\right)$. Adults reported the proactive deck as preferred more than chance $\left(78.67 \%, \chi^{2}=\right.$ $23.52, p<.001)$, 10yo responded at chance $\left(50 \%, \chi^{2}=0, p=\right.$ 1 ), and 5 yo reported marginal preference for the reactive deck $\left(63.41 \%, \chi^{2}=2.44, p=.12\right)$. Adults reported the proactive deck as easier $\left(77.03 \%, \chi^{2}=20.55, p<.001\right), 10$ yo responded at chance levels $\left(40 \%, \chi^{2}=1.23, p=.268\right)$, whereas 5 yo reported the reactive deck as easier $\left(76.19 \%, \chi^{2}=10.5, p<.01\right)$. Adults reported that reactive deck required harder thinking (72.95\%, $\left.\chi^{2}=14.76, p<.001\right)$, whereas the 10 yo and 5 yo did not significantly differ from chance (10yo: $36.84 \%$ selected the reactive deck, $\chi^{2}=2.13 p=.144 ; 5$ yo: $41.46 \%$ selected the reactive deck, $\chi^{2}=0.88, p=.349$ ).

For questions about performance differences between decks, age groups differed in which deck they reported as responding faster $\left(\chi^{2}=11.47, p=.005\right)$ and more accurately $\left(\chi^{2}=15.56, p<.001\right)$ on. Adults responded that they were

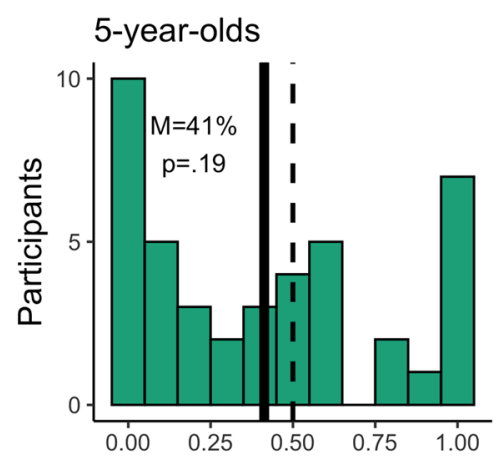

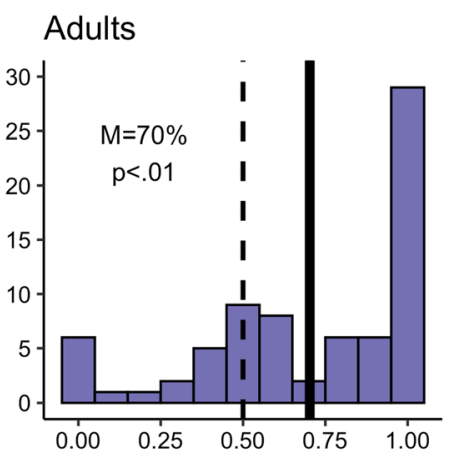

Fig. 2 Histograms of the proportion of proactive deck selections across groups. The dotted black lines indicate chance selections, and the solid black lines indicate group means (5-year-olds: 41.29\%; 10-year-olds:
50.65\%; Adults: $70.27 \%$ ). Proportions of proactive deck selections increased with age, with only adults selecting the proactive deck more than chance 


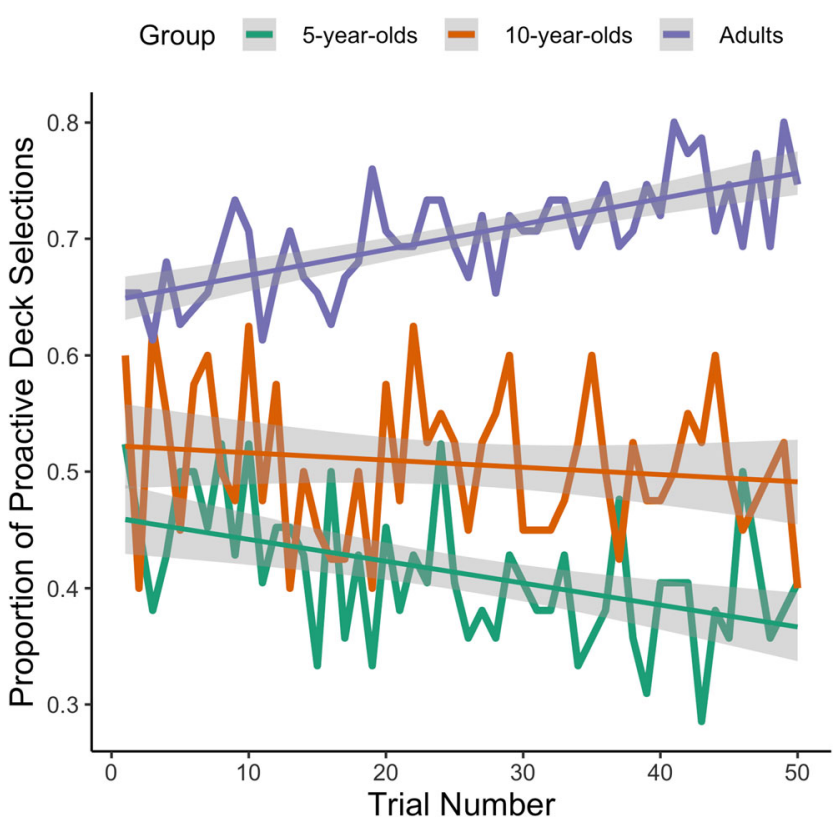

Fig. 3 Trial number predicted proactive deck choices, with proactive selections decreasing across trials. Age group predicted additional variance after controlling for trial number, with older participants making more proactive deck selections. The interaction of age and trial number predicted additional variance in proactive deck choices, reflecting adults increasing their proactive deck selections across trials, 5-year-olds increasing their reactive deck selections across trials, and 10-year-olds showing no significant change across trials faster on the proactive deck $\left(73.61 \%, \chi^{2}=15.13, p<.001\right)$, whereas the child groups did not differ from chance in their responses (10yo: $62.50 \%, \chi^{2}=2.03, p=.155 ; 5$ yo: $41.46 \%$, $\left.\chi^{2}=0.88, p=.349\right)$. Adults reported that they were more accurate on the proactive deck $\left(68.06 \%, \chi^{2}=8.68, p=\right.$ $.003), 10 y o$ responded at chance levels $\left(40 \%, \chi^{2}=1.23, p=\right.$ .268 ), and the 5 yo reported that they were more accurate on the reactive $\operatorname{deck}\left(33.33 \%, \chi^{2}=4.02, p=.045\right)$.

\section{Awareness of task differences predicts preferential task selection in $\mathbf{5 y o}$ and adults}

In a prespecified exploratory analysis, we focused on participants who reported observing deck differences to determine whether individuals who successfully reported task differences systematically selected particular decks. This analysis was conducted to address the heterogeneity in reporting task differences in the 5-year-olds compared with the older age groups, which could confound analyses of deck selections with the full sample. We predicted that in this subset, adults $(N=64)$ and 10yo $(N=33)$ would play the proactive deck, whereas 5 yo $(N=20)$ would play the reactive deck. Age groups significantly differed in deck selections (Kruskal-Wallis chi-squared $=25.78, p<$ $.001)$. Adults played the proactive deck more than 10yo $(p<$ $.001)$ and 5 yo $(p<.001)$, and 10yo showed a trend toward playing the proactive deck more than 5 yo $(p=.06)$, as indicated by follow-up pairwise comparisons using the Wilcoxon rank
5 -year-olds

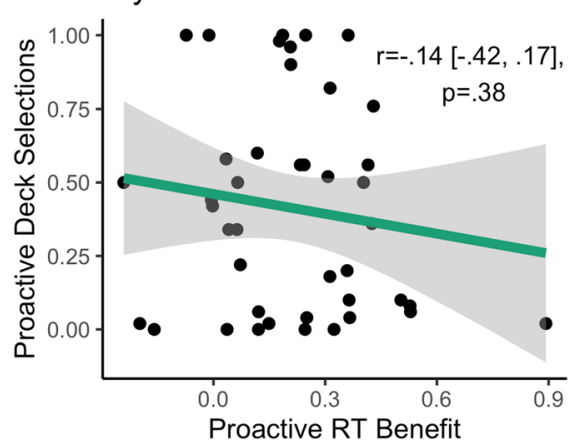

5 -year-olds

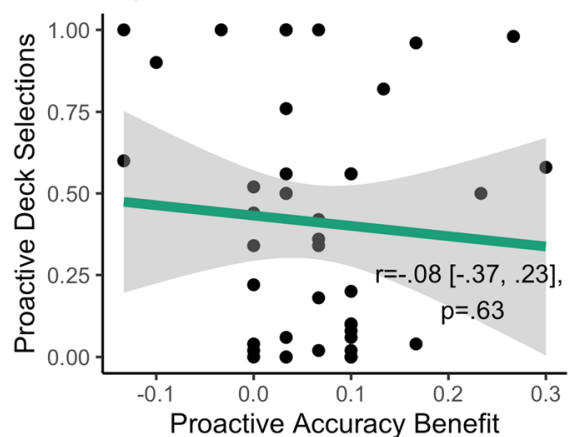

10-year-olds

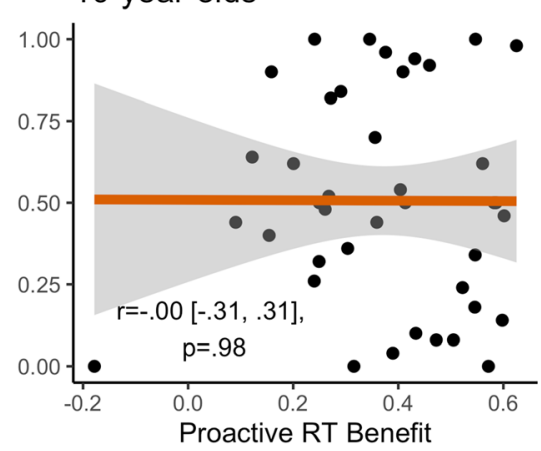

10-year-olds

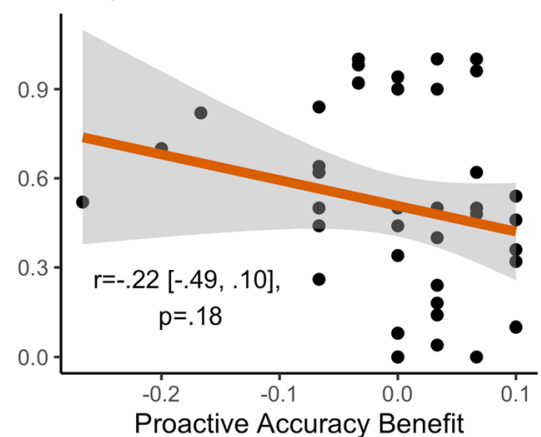

Adults

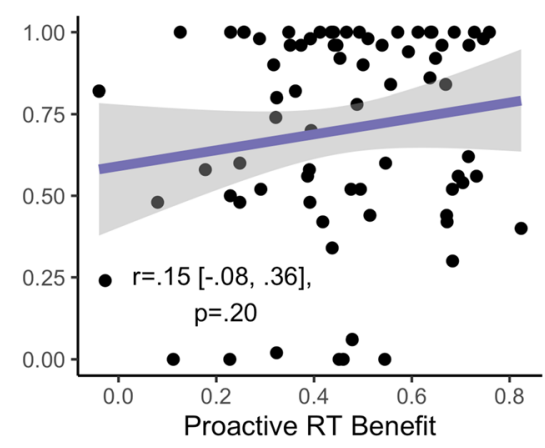

Adults

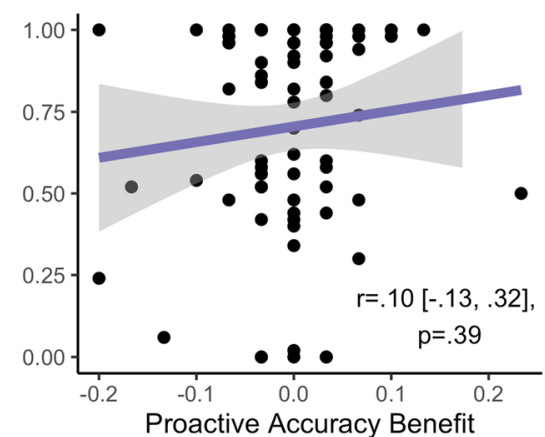

Fig. 4 Across age groups, no significant relationships were observed between proactive deck selections and accuracy or response time differences between proactive and reactive decks during deck familiarization, in contrast with prior findings in the task-switching domain 
Table 2. Proactive deck preferences and awareness of deck and performance differences

\begin{tabular}{|c|c|c|c|}
\hline & $\begin{array}{l}5 \text {-year- } \\
\text { olds }\end{array}$ & $\begin{array}{l}10 \text {-year- } \\
\text { olds }\end{array}$ & Adults \\
\hline Proportion reporting deck differences** & $47.61 \%$ & $82.50 \%{ }^{\wedge \wedge}$ & $86.49 \% \wedge$ \\
\hline Proportion reporting Preference for the proactive deck $* *$ & $36.59 \%$ & $50.00 \%$ & $78.67 \% \wedge$ \\
\hline Proportion reporting the proactive deck as easier* & $23.81 \%^{\wedge}$ & $40.00 \%$ & $77.03 \% \wedge$ \\
\hline Proportion reporting faster responses on the proactive deck* & $41.46 \%$ & $62.50 \%$ & $73.61 \% \wedge$ \\
\hline Proportion reporting better accuracy on the proactive deck** & $33.33 \%^{\wedge}$ & $40.00 \%$ & $68.06 \% \wedge$ \\
\hline Proportion reporting thinking harder on the proactive deck ${ }^{* *}$ & $58.54 \%$ & $63.16 \%$ & $27.03 \% \wedge$ \\
\hline
\end{tabular}

Note. Data are presented as the proportion responses within group to binary posttask questions. $*$ indicates group differences at $p<.01, * * p<.001 . \wedge$ indicates significant differences from chance at $p<.05$ and ${ }^{\wedge}$ at $p<.001$

sum test. As expected, adults chose to play the proactive deck more often than chance $(M=74.09 \%, S D=28.97, p<.001)$; however, 10yo played decks at chance levels (10yo: $M=$ $51.88 \%, S D=30.85, p=.789)$. As predicted, 5 yo chose to play the reactive deck significantly more than chance (5yo: $M=$ $33.20 \%, S D=30.35, p=.022$ ).

We also conducted an exploratory hierarchical model predicting proactive deck selections using a linear code for age group (5-year-olds coded as 0 ), deck awareness (dummy coded as 1: Yes or 0: No for reported differences between decks), and their interaction, with random intercepts and trial slopes for participants. No main effects were observed (age group: $B=-0.04, z=-.19, p=.85$; deck awareness: $B=$ $-1.28, \mathrm{z}=-1.58, p=.12$ ), but a significant age group by deck awareness interaction was observed $(B=0.82, z=2.03, p=$ $.04)$. This interaction reflected the fact that deck awareness was increasingly associated with proactive deck selections with age. These results provide further support that aware 5year-olds are more likely to select the reactive deck and that aware adults are more likely to select the proactive deck.

\section{Discussion}

The present study investigated the development of metacontrol by examining whether children and adults were aware of and selected tasks in response to cognitive demands based on the temporal dynamics of control. Five-year-olds, 10-year-olds, and adults all responded faster on the task option enabling proactive control than the option requiring reactive control, and these relative response time benefits increased with age; only 5-year-olds were less accurate on the proactive deck compared with the reactive deck, whereas the 10-yearolds and adults were similarly accurate across decks. Confirming our hypotheses, proactive deck selections and the percentage of individuals reporting differences between decks increased with age. However, despite clear indices of performance differences across all age groups, only adults preferentially selected the proactive control deck, and only adults and 10-year-olds consistently reported differences between decks. Adults reported better performance on the proactive deck and the proactive deck as preferable and easier, reflecting their deck choices. Although 10-year-olds reported deck differences, they did not systematically report either deck as easier or preferred or report performance differences between decks. Interestingly, 5-year-olds reported the reactive deck as easier and leading to better accuracy, despite not preferentially selecting the reactive deck, not reporting differences between decks, and not expressing a deck preference. However, the subset of 5-year-olds that reported deck differences preferentially played the reactive deck, and 5-year-olds overall selected the reactive deck with increasing frequency as the task progressed. Collectively, these results suggest that younger children are less aware of proactive and reactive control demands between tasks than older children and adults. Further, awareness of task differences leads to different task selections that vary by age, with younger children more likely to select the reactive deck and adults more likely to select the proactive deck.

Performance indices of control demands did not predict deck selections in any age group. One explanation for these null results is the restricted range in the accuracy and response time differences between task options observed in all groups compared with prior investigations (e.g., Kool et al., 2010; Niebaum et al., 2019), which limits statistical power to detect correlations. The difference in response times between decks was also very large for most adults. Thus, the response efficiency demand signal may have reached a minimum threshold to adapt task selection for adults. Further, task performance is typically only weakly predictive of task preferences in older children and adults (Chevalier, 2018; Westbrook, Kester, \& Braver, 2013). Individuals may have developed task preferences utilizing additional factors beyond performance indices of cognitive demand.

Although the 5-year-olds reported better performance and ease on the reactive deck, they did not adapt task selection to maximize accuracy. Young children may not preferentially attend to performance signals to guide behavior, even when 
accurately reporting performance differences between tasks (O’Leary \& Sloutsky, 2019). Young children may also track performance on different tasks without specifically attending to differences between tasks. The by-trial and long-term performance feedback included here may have aided children in monitoring response accuracy, especially for posttask responses. Systematic overestimations of performance accuracy in younger children, even with feedback, may further attenuate demand signals to guide task selection (Lipko, Dunlosky, \& Merriman, 2009; O'Leary \& Sloutsky, 2017, 2019; Schneider, 1998; Yussen \& Berman, 1981).

Older children, who have been shown to avoid unnecessary control demands in a task-switching context, may prioritize accuracy to signal task demands (Niebaum et al., 2019). The accuracy performance feedback used here may have further biased children to attend to accuracy as a demand signal. Thus, the minimal accuracy differences between decks may explain why 10 -year-olds did not preferentially select either deck. However, older children were proficient at reporting task differences, further suggesting that 10 -year-olds successfully monitored task demands but that the relative demands instantiated may have been insufficient to guide task selection. Because proactive control is still improving throughout late childhood into adulthood (Andrews-Hanna et al., 2011; Vink et al., 2014), 10-year-olds may have less facility in engaging control proactively compared with adults, leading to lower preference for the proactive deck compared with adults.

Deciding when and how to implement control may rely on the effective engagement of brain regions supporting cognitive control. Connectivity between $\mathrm{dACC}$ and $\mathrm{PFC}$, regions associated with cognitive control, has been implicated in adaptively selecting tasks to reduce cognitive control demands (McGuire \& Botvinick, 2010; Shenhav, Botvinick, \& Cohen, 2013; Shenhav et al., 2017; Sheth et al., 2012; cf. Sayalı \& Badre, 2019). Connectivity between IPFC and striatum has been further linked to cognitive effort-based decision-making in adults (Botvinick \& Braver, 2015). Because functional connections between $\mathrm{PFC}$ and $\mathrm{dACC}$ and $\mathrm{PFF}$ and striatum increase with age (Ezekiel et al., 2013; Fiske \& Holmboe, 2019; Grayson \& Fair, 2017; Lopez et al., 2019; Luna et al., 2010; Vink et al., 2014), developmental differences in the awareness of control demands and adaptive task selection may reflect age-related differences in the neurological mechanisms supporting these processes.

The high proactive deck preference observed in adults could be viewed as challenging a typical characterization of proactive control as more demanding than reactive control. Specifically, reactive control is characterized by transient activation and recruitment of goal-relevant information rather than sustained activation in lateral prefrontal areas, so proactive control is typically considered more demanding on working memory (Braver, 2012; Marklund \& Persson, 2012). However, resolving response conflict is also demanding, resulting in slower response times and worse accuracy, and is associated with activity in dACC. Adults have been shown to preferentially select tasks with fewer response conflicts if aware of these relative demand differences (Desender, Buc Calderon, Van Opstal, \& Van den Bussche, 2017; Schouppe, Ridderinkhof, Verguts, \& Notebaert, 2014). In the current demand selection task, the conflict between rule cues and targets may have outweighed the effort to engage proactive control, given that the delays were brief and did not include distractors, minimizing demand for sustained representations of rule cues. Moreover, the proactive deck allowed for task preparation, which may have resulted in IPFC activation to bias attention towards only relevant stimuli dimensions (Brass \& von Cramon, 2004). Thus, adults may have experienced attenuated demand signals from $\mathrm{dACC}$ while playing the proactive deck relative to the reactive deck, addition to the improved response efficiency on the proactive deck, which adults may rely on to select tasks (Kool et al., 2010; Niebaum et al., 2019).

Our results suggest that the dramatic developments children show in cognitive control may in part reflect improvements in their metacontrol, that is, an increasing awareness of cognitive task demands and improved ability to adapt control to such demands. Adults and 5-year-olds who reported differences between decks preferentially selected decks that enabled their preferred temporal control modes, with 5-year-olds choosing to play the reactive deck more often and adults choosing to play the proactive deck. Because preferential task selection was specific to the smaller subset of 5-year-olds who reported task differences, metacognitive awareness of deck demands may be requisite for adaptive metacontrol, reflecting prior work in adults (Desender et al., 2017; Gold et al., 2015; cf. O'Leary \& Sloutsky, 2019). Further, only about half of the 5 -year-olds reported deck differences, whereas similar proportions of 10-year-olds and adults reported deck differences, suggesting that children may transition towards spontaneously monitoring cognitive task demands at around 5 years of age.

Although we provide evidence for age-related differences in task selection based on temporal control demands, our study has several limitations. First, our proactive control manipulation was still very short, as participants saw the target directly after the sorting rule. Increasing the duration between rule and target presentation, making proactive control more difficult, could make adults and older children prefer the reactive task. Our use of accuracy performance feedback could also influence task selections. Including response time feedback may bias individuals towards assessing demand via response efficiency, resulting in greater preference for the proactive task option. Removing feedback could also hinder individuals' ability to assess demand, especially children (e.g., O'Leary \& Sloutsky, 2019). Although the posttask questionnaire yielded insight into age-related differences in awareness of task differences and preferences, young children also likely 
have limited ability to verbally report metacognitive knowledge compared with older children and adults, which may have influenced these analyses. Further, we are unable to confidently discern whether adults' proactive task preferences were due to improving response efficiency or avoiding conflict, or both, from only posttask questioning.

Additional research should investigate whether individuals monitor different signals of demand at different ages and how these signals influence task decisions and lead to potential benefits across development. Young children may be less likely to utilize signals of cognitive demands for guiding behavior relative to adults (Niebaum et al., 2019; O'Leary \& Sloutsky, 2017); they may prioritize other signals such as novelty and interest when making task selections, which may benefit their learning. Developmental improvements in selecting tasks based on demand could also reflect faster learning of task demands with age. Understanding of cognitive development may thus be advanced by incorporating considerations of how and when children attend to signals of control demand, the different contexts in which children decide to engage control, the cognitive abilities supporting demand monitoring and adaptive task selections, and how these factors influence children's choices and outcomes.

Acknowledgements The authors thank Shadi Sharif, Jennifer Felker, Hayden Morano, Samantha Bartolo, and Grace Dostart for assistance in participant recruitment and data collection and Rebecca Wu, Benjamin Schenkel, and Hayden Morano for coding response data.

Funding This work was supported by the National Institutes of Health (R01 HD086184-02) and a National Science Foundation Graduate Research Fellowship to J.C.N. (Grant No. 1650042).

\section{References}

Ahmed, S. F., Tang, S., Waters, N. E., \& Davis-Kean, P. (2019). Executive function and academic achievement: Longitudinal relations from early childhood to adolescence. Journal of Educational Psychology, 111(3), 446.

Andrews-Hanna, J. R., Seghete, K. L. M., Claus, E. D., Burgess, G. C., Ruzic, L., \& Banich, M. T. (2011). Cognitive control in adolescence: Neural underpinnings and relation to self-report behaviors. PLOS ONE, 6(6).

Best, J. R., Miller, P. H., \& Naglieri, J. A. (2011). Relations between executive function and academic achievement from ages 5 to 17 in a large, representative national sample. Learning and Individual Differences, 21(4), 327-336.

Blair, C., \& Razza, R. P. (2007). Relating effortful control, executive function, and false belief understanding to emerging math and literacy ability in kindergarten. Child Development, 78(2), 647-663.

Botvinick, M., \& Braver, T. (2015). Motivation and cognitive control: from behavior to neural mechanism. Annual Review of Psychology, 66.

Brass, M., \& von Cramon, D. Y. (2004). Decomposing components of task preparation with functional magnetic resonance imaging. Journal of Cognitive Neuroscience, 16(4), 609-620.

Braver, T. S. (2012). The variable nature of cognitive control: a dual mechanisms framework. Trends in Cognitive Sciences, 16(2), 106113.
Buss, A. T., \& Spencer, J. P. (2018). Changes in frontal and posterior cortical activity underlie the early emergence of executive function. Developmental Science, 21(4), e12602.

Carlson, S. M., \& Wang, T. S. (2007). Inhibitory control and emotion regulation in preschool children. Cognitive Development, 22(4), 489-510.

Cartwright, K. B. (2012). Insights from cognitive neuroscience: The importance of executive function for early reading development and education. Early Education \& Development, 23(1), 24-36.

Chatham, C. H., Frank, M. J., \& Munakata, Y. (2009). Pupillometric and behavioral markers of a developmental shift in the temporal dynamics of cognitive control. Proceedings of the National Academy of Sciences of the United States of America, 106(14), 5529-5533.

Chevalier, N. (2015). The development of executive function: Toward more optimal coordination of control with age. Child Development Perspectives, 9(4), 239-244.

Chevalier, N. (2018). Willing to think hard? The subjective value of cognitive effort in children. Child Development, 89(4), 1283-1295.

Chevalier, N., Dauvier, B., \& Blaye, A. (2018). From prioritizing objects to prioritizing cues: a developmental shift for cognitive control. Developmental science, 21(2), e12534.

Chevalier, N., Martis, S. B., Curran, T., \& Munakata, Y. (2015). Metacognitive processes in executive control development: The case of reactive and proactive control. Journal of Cognitive Neuroscience, 27(6), 1125-1136.

Davidson, M. C., Amso, D., Anderson, L. C., \& Diamond, A. (2006). Development of cognitive control and executive functions from 4 to 13 years: Evidence from manipulations of memory, inhibition, and task switching. Neuropsychologia, 44(11), 2037-2078.

Desender, K., Buc Calderon, C., Van Opstal, F., \& Van den Bussche, E. (2017). Avoiding the conflict: Metacognitive awareness drives the selection of low-demand contexts. Journal of Experimental Psychology: Human Perception and Performance, 43(7), 1397.

Doebel, S., Barker, J. E., Chevalier, N., Michaelson, L. E., Fisher, A. V., \& Munakata, Y. (2017). Getting ready to use control: Advances in the measurement of young children's use of proactive control. PLOS $O N E, 12(4)$.

Enkavi, A. Z., Eisenberg, I. W., Bissett, P. G., Mazza, G. L., MacKinnon, D. P., Marsch, L. A., \& Poldrack, R. A. (2019). Large-scale analysis of test-retest reliabilities of self-regulation measures. Proceedings of the National Academy of Sciences, 116(12), 5472-5477.

Ezekiel, F., Bosma, R., \& Morton, J. B. (2013). Dimensional change card sort performance associated with age-related differences in functional connectivity of lateral prefrontal cortex. Developmental Cognitive Neuroscience, 5, 40-50.

Faul, F., Erdfelder, E., Lang, A. G., \& Buchner, A. (2007). G* Power 3: A flexible statistical power analysis program for the social, behavioral, and biomedical sciences. Behavior Research Methods, 39(2), 175191.

Fiske, A., \& Holmboe, K. (2019). Neural substrates of early executive function development. Developmental Review, 52, 42-62.

Gathercole, S. E., Pickering, S. J., Ambridge, B., \& Wearing, H. (2004). The structure of working memory from 4 to 15 years of age. Developmental Psychology, 40(2), 177.

Gold, J. M., Kool, W., Botvinick, M. M., Hubzin, L., August, S., \& Waltz, J. A. (2015). Cognitive effort avoidance and detection in people with schizophrenia. Cognitive, Affective, and Behavioral Neuroscience, 15(1), 145-154.

Gonthier, C., Zira, M., Colé, P., \& Blaye, A. (2019). Evidencing the developmental shift from reactive to proactive control in early childhood and its relationship to working memory. Journal of Experimental Child Psychology, 177, 1-16.

Grayson, D. S., \& Fair, D. A. (2017). Development of large-scale functional networks from birth to adulthood: A guide to the neuroimaging literature. NeuroImage, 160, 15-31. 
Koechlin, E., \& Summerfield, C. (2007). An information theoretical approach to prefrontal executive function. Trends in Cognitive Sciences, 11(6), 229-235.

Kool, W., McGuire, J. T., Rosen, Z. B., \& Botvinick, M. M. (2010). Decision making and the avoidance of cognitive demand. Journal of Experimental Psychology: General, 139(4), 665.

Lipko, A. R., Dunlosky, J., \& Merriman, W. E. (2009). Persistent overconfidence despite practice: The role of task experience in preschoolers' recall predictions. Journal of Experimental Child Psychology, 103(2), 152-166.

Lopez, K. C., Kandala, S., Marek, S., \& Barch, D. M. (2019). Development of network topology and functional connectivity of the prefrontal cortex. Cerebral Cortex, 30(4), 2489-2505. doi: https://doi.org/10.1093/cercor/bhz255

Lucenet, J., \& Blaye, A. (2014). Age-related changes in the temporal dynamics of executive control: A study in 5-and 6-year-old children. Frontiers in Psychology, 5, 831.

Luna, B., Padmanabhan, A., \& O'Hearn, K. (2010). What has fMRI told us about the development of cognitive control through adolescence?. Brain and Cognition, 72(1), 101-113.

Makowski, D., Ben-Shachar, M., \& Lüdecke, D. (2019). bayestestR: Describing effects and their uncertainty, existence and significance within the Bayesian framework. Journal of Open Source Software, 4(40), 1541.

Marklund, P., \& Persson, J. (2012). Context-dependent switching between proactive and reactive working memory control mechanisms in the right inferior frontal gyrus. NeuroImage, 63(3), 1552-1560.

McDonald, J. H. (2014). Kruskal-Wallis test. Handbook of Biological Statistics (pp. 157-164). Retrieved from http://www. biostathandbook.com/kruskalwallis.html

McGuire, J. T., \& Botvinick, M. M. (2010). Prefrontal cortex, cognitive control, and the registration of decision costs. Proceedings of the National Academy of Sciences, 107(17), 7922-7926.

McHugh, M. L. (2013). The chi-square test of independence. Biochemia Medica: Biochemia Medica, 23(2), 143-149.

Meiran, N. (1996). Reconfiguration of processing mode prior to task performance. Journal of Experimental Psychology: Learning, Memory, and Cognition, 22(6), 1423.

Meyer, J. P., \& Seaman, M. A. (2013). A comparison of the exact Kruskal-Wallis distribution to asymptotic approximations for all sample sizes up to 105. The Journal of Experimental Education, $81(2), 139-156$.

Moffitt, T. E., Arseneault, L., Belsky, D., Dickson, N., Hancox, R. J., Harrington, H., ... Sears, M. R. (2011). A gradient of childhood selfcontrol predicts health, wealth, and public safety. Proceedings of the National Academy of Sciences of the United States of America, 108(7), 2693-2698.

Monsell, S. (2003). Task switching. Trends in Cognitive Sciences, 7(3), 134-140.

Morey, R. D., Rouder, J. N., Jamil, T., \& Morey, M. R. D. (2015). Package 'bayesfactor' [Computer software].

Munakata, Y., Snyder, H. R., \& Chatham, C. H. (2012). Developing cognitive control: Three key transitions. Current Directions in Psychological Science, 21(2), 71-77.

Niebaum, J. C., Chevalier, N., Guild, R. M., \& Munakata, Y. (2019). Adaptive control and the avoidance of cognitive control demands across development. Neuropsychologia, 123, 152-158.

Niendam, T. A., Laird, A. R., Ray, K. L., Dean, Y. M., Glahn, D. C., \& Carter, C. S. (2012). Meta-analytic evidence for a superordinate cognitive control network subserving diverse executive functions. Cognitive, Affective, and Behavioral Neuroscience, 12(2), 241-268.

O'Leary, A. P., \& Sloutsky, V. M. (2017). Carving metacognition at its joints: Protracted development of component processes. Child Development, 88(3), 1015-1032.
O’Leary, A. P., \& Sloutsky, V. M. (2019). Components of metacognition can function independently across development. Developmental Psychology, 55(2), 315.

Peirce, J., Gray, J. R., Simpson, S., MacAskill, M., Höchenberger, R., Sogo, H., . . . Lindeløv, J. K. (2019). PsychoPy2: Experiments in behavior made easy. Behavior Research Methods, 51(1), 195-203.

Prencipe, A., Kesek, A., Cohen, J., Lamm, C., Lewis, M. D., \& Zelazo, P. D. (2011). Development of hot and cool executive function during the transition to adolescence. Journal of Experimental Child Psychology, 108(3), 621-637.

Robson, D. A., Allen, M. S., \& Howard, S. J. (2020). Self-regulation in childhood as a predictor of future outcomes: A meta-analytic review. Psychological Bulletin, 146(4), 324-354. doi:https://doi.org/10. 1037/bul0000227

RStudio Team. (2015). RStudio: Integrated development for R. Boston, MA: RStudio, Inc. Retrieved from http://www.rstudio.com

Sayalı, C., \& Badre, D. (2019). Neural systems of cognitive demand avoidance. Neuropsychologia, 123, 41-54.

Schneider, W. (1998). Performance prediction in young children: Effects of skill, metacognition and wishful thinking. Developmental Science, 1(2), 291-297.

Schouppe, N., Ridderinkhof, K. R., Verguts, T., \& Notebaert, W. (2014). Context-specific control and context selection in conflict tasks. Acta Psychologica, 146, 63-66.

Shenhav, A., Botvinick, M. M., \& Cohen, J. D. (2013). The expected value of control: An integrative theory of anterior cingulate cortex function. Neuron, 79(2), 217-240.

Shenhav, A., Musslick, S., Lieder, F., Kool, W., Griffiths, T. L., Cohen, J. D., \& Botvinick, M. M. (2017). Toward a rational and mechanistic account of mental effort. Annual Review of Neuroscience, 40, 99 124.

Sheth, S. A., Mian, M. K., Patel, S. R., Asaad, W. F., Williams, Z. M., Dougherty, D. D., ... Eskandar, E. N. (2012). Human dorsal anterior cingulate cortex neurons mediate ongoing behavioural adaptation. Nature, 488(7410), 218.

Troller-Renfree, S. V., Buzzell, G. A., \& Fox, N. A. (2020). Changes in working memory influence the transition from reactive to proactive cognitive control during childhood. Developmental Science, e12959.

Vink, M., Zandbelt, B. B., Gladwin, T., Hillegers, M., Hoogendam, J. M., van den Wildenberg, W. P., ... Kahn, R. S. (2014). Frontostriatal activity and connectivity increase during proactive inhibition across adolescence and early adulthood. Human Brain Mapping, 35(9), 4415-4427.

Wendelken, C., Munakata, Y., Baym, C., Souza, M., \& Bunge, S. A. (2012). Flexible rule use: Common neural substrates in children and adults. Developmental Cognitive Neuroscience, 2(3), 329-339.

Westbrook, A., Kester, D., \& Braver, T. S. (2013). What is the subjective cost of cognitive effort? Load, trait, and aging effects revealed by economic preference. PLOS ONE, 8(7).

Wickham, H. (2016). ggplot2: elegant graphics for data analysis. Springer.

Yussen, S. R., \& Berman, L. (1981). Memory predictions for recall and recognition in first-, third-, and fifth-grade children. Developmental Psychology, 17(2), 224.

Zelazo, P. D., \& Carlson, S. M. (2012). Hot and cool executive function in childhood and adolescence: Development and plasticity. Child Development Perspectives, 6(4), 354-360.

Publisher's note Springer Nature remains neutral with regard to jurisdictional claims in published maps and institutional affiliations. 